\title{
Trauma Por Onda Explosiva La Mirada Del Cirujano General En La Práctica Civil
}

\author{
Sebastian Sanchez ${ }^{1}$, Mauricio Pedraza ${ }^{2}$, Luis F Cabrera ${ }^{3}$, Daniel Gomez ${ }^{4}$, Andres Mendoza ${ }^{5}$, Jean A Pulido ${ }^{6}$, Juliana Ordoñez ${ }^{7}$
}

\section{Resumen}

Introducción: Onda explosiva se define como la liberación violenta de energía que es transferida hacia el ambiente; siendo esta capaz de empujar columnas de aire, material particulado y gases calientes que al encontrarse con el cuerpo humano ocasionan lesiones que pueden llegar a comprometer la vida.

Materiales y métodos: Se presenta una revisión narrativa de la literatura con herramientas de revisión sistemática sobre el tema de trauma por onda expansiva. Excluyendo artículos no relacionados con trauma por onda expansiva publicados en idiomas diferentes al español y al inglés y aquellos cuya temática central es diferente de lo tratado por la cirugía general y sus subespecialidades.

Discussion: Se describe cada uno de los 5 mecanismos de lesión descritos para este tipo de trauma, y se hace un resumen del diagnóstico y manejo de las lesiones más frecuentes a nivel torácico, abdominal y vascular periférico.

Conclusión: Es esencial conocer los mecanismos de lesión para dar el manejo adecuado a los pacientes víctimas de este tipo de lesiones de una manera multidisciplinaria.

Palabras clave: Cirugía general, Onda explosiva, Poli-trauma, Terrorismo, Trauma, Trauma de explosión.

\begin{abstract} particulate material and hot gases that when encountering the human body can cause potentially mortal injuries. management of the most frequent thoracic, abdominal and peripheral vascular injuries associated to blast injury. organised and global manner.

Keywords: Blast injury, Blast trauma, General surgery, Polytrauma, Terrorism, Trauma.

Panamerican Journal of Trauma, Critical Care \& Emergency Surgery (2019): 10.5005/jp-journals-10030-1249
\end{abstract}

Introduction: Explosive blast is defined as the violent energy release that is transferred to the environment; being able to push columns of air,

Materials and methods: A narrative review about blast trauma is presented. Articles not related to blast trauma published in languages other than Spanish and English and those whose central theme was different from that is treated by general surgery and its subspecialties were excluded. Discussion: Each of the 5 injury mechanisms described for this type of trauma is described, and a summary is made of the diagnosis and

Conclusion: It is essential to know the mechanisms of injury to give the appropriate management to the victims of this type of injuries in a

\section{INTRODUCCIÓN}

"En memoria de las víctimas del atentado terrorista del 17 de enero de 2019 en las instalaciones de Escuela de Policía General Santander, en Bogotá, Colombia."

Onda explosiva se define como la liberación violenta de energía que es transferida hacia el ambiente; siendo esta capaz de empujar columnas de aire, material particulado y gases calientes producto de la detonación que al encontrarse con el cuerpo humano ocasionan lesiones contusas, penetrantes o termoquímicas entre otras. ${ }^{1,2}$

Para el 2000 y el 2005, Camargo et al. reportan que el $80 \%$ de las heridas de miembros de las Fuerzas Militares de Colombia eran causadas por arma de fuego de alto calibre y velocidad. En esa época, la guerra se hacía en confrontación directa y con armamento convencional. No obstante, la técnica de guerra fue cambiando y se evitaba el combate directo y, entre 2009 y 2010, el $75 \%$ de las heridas se debieron a ex-plosivos no convencionales y bombas antipersonales. Es por esto que la epidemiología de las heridas varió y en los últimos años los pacientes sufren lesiones con una gran pérdida de tejido, con gran potencial de infección, y con una alta tasa de amputaciones y desmembramientos. ${ }^{3}$
${ }^{1}$ Residente Cirugía General, Pontificia Universidad Javeriana, Bogota, Colombia

${ }^{2}$ Residente Cirugía General, Universidad El Bosque, Bogotá, Colombia

${ }^{3}$ Cirujano General, Universidad El Bosque, Fundacion Santa Fe de Bogotá, Bogotá, Colombia

${ }^{4}$ Cirugano General Universidad Militar Central, Bogotá, Colombia

${ }^{5}$ Cirujano General Universidad El Bosque, Bogotá, Colombia

${ }^{6}$ Estudiante de Medicina, Universidad El Bosque, Bogotá, Colombia

${ }^{7}$ Departamento Cirugía de Trauma, Fundación valle de lili, Cali, Colombia

Corresponding Author: Mauricio Pedraza, Residente Cirugía General, Universidad El Bosque, Bogotá, Colombia, Phone: +57 (301)3709557, e-mail: mpedraza93@gmail.com

How to cite this article: Sanchez S, Pedraza M, Cabrera LF, et al. Trauma Por Onda Explosiva La Mirada Del Cirujano General En La Práctica Civil. Panam J Trauma Crit Care Emerg Surg 2019;8(3):180-186.

Source of support: Nil

Conflict of interest: None

() The Author(s). 2019 Open Access This article is distributed under the terms of the Creative Commons Attribution 4.0 International License (https://creativecommons. org/licenses/by-nc/4.0/), which permits unrestricted use, distribution, and non-commercial reproduction in any medium, provided you give appropriate credit to the original author(s) and the source, provide a link to the Creative Commons license, and indicate if changes were made. The Creative Commons Public Domain Dedication waiver (http://creativecommons.org/publicdomain/zero/1.0/) applies to the data made available in this article, unless otherwise stated. 
El estudio del trauma por onda explosiva cobra una real importancia por el hecho de que los explosivos ocasionan entre el 66 al 84\% de las lesiones en combate según cifras de los conflictos en Iraky Afganistán. ${ }^{4-6}$ Dichas lesiones comprometen en la mayoría de los casos, 2 o más sistemas por lo que requieren de un manejo multidisciplinario. Adicionalmente es esencial mencionar que las heridas por onda explosiva no se limitan al contexto bélico; ya que en la actualidad, la población civil también se encuentra expuesta a posibles lesiones de este tipo por ataques terroristas, accidentes relacionados con la industria, el hogary otros. ${ }^{1,7,8}$ Un ejemplo de ello es el ataque terrorista del 17 de enero de 2019 en las instalaciones de la Escuela de Policía General Santander, en Bogotá, Colombia, un donde un automóvil cargado con explosivos dejó un saldo de 22 muertos y 69 heridos. Por lo anterior el trauma por onda explosiva no debe considerarse una entidad de manejo exclusivo por parte del cirujano de guerra, por el contrario, todo cirujano de práctica civil debe conocer los patrones y mecanismos de lesión asociados a las explosiones para asegurar el manejo apropiado de estos pacientes. ${ }^{2,6,8}$

Es por todo lo expuesto con anterioridad que los autores nos permitimos presentar una revisión crítica de la literatura, en honor a las víctimas del atentado antes mencionado, con el objetivo de que le sirva al lector como herramienta de estudio sobre el trauma por onda explosiva partiendo desde la mirada de un cirujano general en la práctica civil permitiéndole abordar estos pacientes de una manera integral y adecuada.

\section{Materiales y Métodos}

Se presenta una revisión narrativa de la literatura con herramientas de revisión sistemática sobre el tema de trauma por onda expansiva. La búsqueda de material bibliográfico fue realizada en la base de datos de PubMed generando un algoritmo de búsqueda con las palabras "Blast injury", "Blast trauma" y "Surgery" teniendo en cuenta artículos publicados en los idiomas español o inglés, durante los últimos 5 años. Fueron excluidos todos aquellos artículos no relacionados con trauma por onda expansiva publicados en idiomas diferentes al español y al inglés y aquellos cuya temática central se sale de lo tratado por la cirugía general y sus subespecialidades. Se completa la búsqueda bibliográfica con artículos clásicos sobre el tema en cuestión y otros recomendados por expertos en el tema.

\section{Discussion}

Estudios recientes han podido identificar un patrón de lesión por onda explosiva que requiere un abordaje específico como lo reportan Popivanov et al. en su experiencia como cirujanos de combate en Afganistán durante 2010. ${ }^{4}$

El trauma por explosivos se asocia a un injury severity score (ISS) alto, mayor tiempo de estancia hospitalaria, peores desenlaces y un mayor número de estructuras comprometidas cuando se le compara a otros tipos de trauma. ${ }^{4,6,9}$ El daño alcanzado depende de múltiples variables Entre las cuales se encuentran distancia al lugar de detonación, entorno (abierto o cerrado, rodeado de agua o aire, etc.), tipo de explosivo y tiempo de exposición entre otros. ${ }^{10}$

En orden descendente los segmentos corporales más frecuentemente comprometidos en explosiones son las extremidades (85.4\%), el tórax (38.2\%), la cabeza (27.2\%), el abdomen (25\%), y la cara (20\%). ${ }^{1,4,5}$ En Colombia, las tres primeras causas de mortalidad inmediata en el campo de batalla, son las heridas de tórax (34\%), el desmembramiento por minas antipersonales
(31\%) y las heridas de cráneo (17\%), segun muestra Camargo et al., ${ }^{3 .}$ sin embargoestas cifras aun no estan actualizadas para este ultimo año. Acorde a lo expuesto previamente, un estudio sobre las lesiones en la población civil durante la guerra en Siria reporta que del total de lesiones el $34.4 \%$ fueron asociadas a explosivos, comprometiendo con más frecuencia las extremidades (56\%), cabeza (20\%) y abdomen (16.2\%). ${ }^{11}$ Desde enero de 1999 hasta diciembre de 2010, el Hospital Militar Central atendió más de 15.000 heridos en combate, 4.233 de ellos críticamente lesionados (ISS > 15), segun lo publicado en la Revista de cirugia general colombiana en 2014 (36), donde se encontr que la mortalidad hospitalaria disminuyó progresivamente de 5,2\% al 2,3\% gracias al alto de grado de conocimientos teóricos y prácticos en la atención del paciente traumatizado, un enfoque multidisciplinario. ${ }^{3}$

Existen diferentes tipos de explosivos; Aquellos diseñados para causar un rápido ascenso de presión, otros diseñados para dañar por conflagración y otros utilizados para propulsar objetos capaces de causar lesiones penetrantes. ${ }^{1}$ Clásicamente se han descrito 5 mecanismos de daño que usualmente se sobreponen; estos son: diferencia de presión (primario), fragmentación (secundario), colapso de estructuras (terciario), calor (cuaternario), infección y radiación (quinario). ${ }^{1,2,4,7}$ A continuación se describe cada uno de ellos.

\section{Mecanismo Primario}

El primer mecanismo está relacionado con el cambio abrupto de presión propagado por el medio; puede comprometer todo tipo de órganos pero presenta especial tropismo por los órganos que contienen aire. Esto se debe a que los bolsillos de aire se comprimen para posteriormente expandirse ocasionando grandes cantidades de energía cinética y daño por medio de implosión. El ejemplo clásico es el compromiso de la membrana timpánica que cede ante un cambio abrupto de presión. ${ }^{1,2,5,10}$ Otro ejemplo de ello es lo que ocurre en el pulmón en donde al implosionar el alveolo puede llevar a entidades como neumotórax, hemotórax, contusión pulmonar, y neumomediastino. ${ }^{1,4}$ A nivel abdominal es posible evidenciar la perforación espontánea, aunque rara (incidencia de 1.2\% a 1.7\%), ha sido descrita en la literatura médica teniendo como factor de riesgo la presencia de hematomas murales, ocasionados por el desgarro de la pared intestinal asociado al mecanismo primario. A pesar de su baja incidencia es necesario sospechar en todo paciente perforacion de viscera hueca ya que de no diagnosticarse puede llevar a alta mortalidad. ${ }^{5}$ El órgano que se perfora con mayor frecuencia es el colon. Las perforaciones previamente mencionadas pueden darse hasta 14 días después del evento lesivo siendo más frecuentes entre los días 3 a 5. ${ }^{1,2,4,5}$ Las perforaciones tardías tienen una incidencia reportada en la literatura de hasta $3 \% .^{5}$ La incidencia del trauma primario abdominal por onda explosiva ha sido reportada de entre el 1.3 al 33\% de los casos según la literatura consultada; sin embargo, es necesario mencionar que se ha visto una incidencia menor cuando se le compara con el compromiso del tórax y del oído., ${ }^{1,4}$ La supervivencia de paciente con trauma abdominal por mecanismo primario de onda expansiva oscila entre el 5.6 y $6.7 \%$. Siendo menor esta menor cuando se trata de explosiones en entornos cerrados, o bajo el agua; esto se debe a que el agua es mejor conductor de energía y a que en espacios cerrados la onda explosiva choca contra muros, transferido más energía sobre los cuerpos presentes. ${ }^{2,10}$ Esto es útil saberlo ya que en el escenario de un ataque a un buzo que está completamente sumergido tiene mayor posibilidad de una lesión potencialmente mortal cuando se 
compara con un buzo que se encuentre apoyado en un dispositivo de flotación que mantiene el tórax fuera del agua. Esto ha sido replicado en modelos animales como el publicado por Han et al. en 2013. 2,12 Teniendo en cuenta lo anterior, el hecho de conocer la cinemática de trauma le sirve al cirujano para tomar decisiones más apropiadas para cada paciente. ${ }^{2}$

\section{Mecanismo Secundario}

Son las lesiones más frecuentes y según la literatura están presentes hasta en el $90 \%$ de los eventos traumáticos asociados a explosión. ${ }^{1,4}$ El $94.4 \%$ de las perforaciones intestinales en trauma por explosiones está relacionado con este mecanismo. ${ }^{5}$

Ocurre cuando la onda expansiva eleva material inerte que acelera hacia un cuerpo causando lesiones penetrantes. ${ }^{1,4,5,10}$ Entre los objetos que pueden ser despedidos por la onda explosiva están: balines, clavos, tornillos, piedras, vidrios, y materia fecal entre otros. ${ }^{4}$ Estos son capaces de atravesar distancias superiores a las alcanzadas por la onda expansiva. ${ }^{10}$ Un ejemplo de lo anterior es el publicado por Ramman et al. quienes reportan un caso de un paciente con antecedente de trauma por onda expansiva quien consultó por persistencia de dolor y signos de infección local a nivel del pubis, al realizar estudios complementarios se logró evidenciar la presencia de un tornillo alojado en la sínfisis púbica, un hallazgo inadvertido durante la intervención inicial. ${ }^{13}$

Este tipo de lesiones cuando comprometen cabeza, cuello y tórax con frecuencia son causales de mortalidad, pues se relacionan con decapitación y amputacion traumatica de extremidades con el compromiso vascular subsecuente. Los objetos que penetran el cuerpo pueden causar lesiones por onda expansiva dentro del tejido mismo a medida que avanzan; esta lesión puede llegar a comprometer 25 veces el diámetro del cuerpo penetrante., ${ }^{1,9}$

En este escenario el medio de propagación también se hace relevante ya que los cuerpos inertes propulsados a gran velocidad en una explosión terrestre no se comportan de la misma manera bajo el agua; esto se debe a la mayor densidad del agua que impide el libre desplazamiento de material a través de ella. 2,12

\section{Mecanismo Terciario}

Corresponde a colapso estructural, el desplazamiento forzado de la víctima, fuerzas de aceleración, desaceleración, y atrapamiento. Usualmente mediante este mecanismo se compromete la integridad de más de un individuo., ${ }^{1,5,10}$ Un ejemplo de ello es el atentado contra el edificio del Departamento Administrativo de Seguridad (DAS) el 6 de diciembre de 1989 en Bogotá, Colombia cuando un bus cargado con aproximadamente 500 kilos de dinamita llevó al colapso de estructuras y causó la muerte 60 personas y dejando aproximadamente 600 heridos.

Asociado al mecanismo terciario se encuentra el trauma por aplastamiento con la rabdomiolisis subsecuente que puede llevar a injuria renal aguda y muerte. ${ }^{1,5}$ También se asocia a trauma craneoencefálico, trauma contuso de tórax y abdomen, y fracturas de huesos largos. ${ }^{4}$

\section{Mecanismos Cuaternario y Quinario}

Los mecanismos cuaternario y quinario hacen referencia a las lesiones térmicas, químicas, ambientales y por radiación. En estos mecanismos también se incluyen las llamadas "bombas sucias" en donde se utiliza material contaminado para causar además de lesión mecánica, un riesgo biológico para la víctima. ${ }^{1,5}$ La sobreinfección de heridas por onda expansiva ocurre hasta en el $10.5 \%$ de los casos. ${ }^{4,10}$

\section{Diagnóstico y Tratamiento}

En la escena de los hechos debe tenerse en cuenta siempre que puede haber un segundo dispositivo explosivo lo cual es esencial asegurar el espacio antes de brindar una atención con el objetivo de proteger al personal de salud y no desperdiciar la oportunidad que tienen los afectados de sobrevivir.'

El correcto triage en el contexto de explosiones es determinante para la sobrevida de los involucrados. Este sistema ha demostrado que al ser realizado por una persona experta permite de manera organizada priorizar la atención a aquellas víctimas que tienen mayor probabilidad de supervivencia., ${ }^{1,8}$

Es necesario entender que los pacientes expuestos a explosiones deben ser abordados de manera integral por un equipo multidisciplinario pensando en que todos los sistemas podrían estar comprometidos. ${ }^{1,13-15}$

El abordaje inicial debe hacerse de manera secuencial y ordenada asegurando lo antes posible la vía aérea llevando a una ventilación efectiva y adecuada siempre teniendo en cuenta la posibilidad de inhalación de gases calientes con el compromiso aéreo subsecuente. Posterior a ello debe protegerse la columna cervical ya que movimientos de aceleración y desaceleración pueden causar fracturas que de desplazarse comprometen estructuras neurológicas de manera irreversible. No debe continuar el manejo específico de lesiones sin antes identificar y tratar las entidades que pueden comprometer la vida del paciente; de allí que la evaluación del cuello y tórax, deben hacerse de manera meticulosa y ordenada. Una de las principales causas de mortalidad es la pérdida de volumen circulante por lo que en la siguiente instancia se debe asegurar una adecuada reposición de volumen por 2 vías periféricas con catéteres de gran calibre; en caso de contar con reservas de hemoderivados se debe iniciar una transfusión masiva de reanimación con esquemas 1 a 1 ya que ha demostrado mejorar la sobrevida de los pacientes y un cierre abdominal temprano en escenarios de guerra como lo describen Glaser et al. ${ }^{1,8,16}$ La víctima debe ser expuesta completamente con el objetivo de realizar un examen físico completo que permita identificar otras lesiones. ${ }^{1,8,10}$ Hakimoglu et al. a partir de su experiencia reconocen las lesiones que comprometen, cabeza, cuello, abdomen y el trauma vascular, como predictores de alta mortalidad por lo que la valoración de estas estructuras debe ser impecable. ${ }^{11}$ La inspección de la membrana timpánica nunca debe obviarse ya que de mostrarse comprometida podría indicar compromiso de estructuras como pulmón, cerebro o vísceras huecas. ${ }^{1,8,10} \mathrm{~A}$ pesar de ello no ha demostrado ser útil como herramienta para establecer el triage como se pensaba con anterioridad. ${ }^{2}$

El uso de medios diagnósticos especializados en el escenario de múltiples lesionados puede llevar a un cuello de botella por lo cual se debe estimar qué pacientes se benefician más que otros ante la realización de estudios paraclínicos por medio de la adecuada evaluación clínica y el análisis crítico de cada caso. ${ }^{8}$ Un ejemplo de ello es que estudios recientes no han encontrado evidencia de que la realización de tomografía de rutina mejore la sobrevida en pacientes con traumas contusos de alto impacto. ${ }^{17}$ Es por ello que algunos autores abogan por el manejo expectante incluso en escenarios de trauma penetrante de abdomen en pacientes 
hemodinámicamente estables sin signos de irritación peritoneal; el manejo expectante en algunos escenarios podría reducir tiempos de estancia hospitalaria, costos, uso de salas de cirugía e insumos y número el de laparotomías no terapéuticas aspectos que cobran importancia cuando hay múltiples heridos. ${ }^{18}$

Algunos estudios han demostrado que el uso temprano de analgesicos fuertes como los opioides se correlacionen con menor incidencia de síndrome de estrés postraumático. ${ }^{1}$

A nivel torácico la lesión pulmonar es una de las más comunes en estos pacientes, y debe sospecharse en todo aquel que presente un descenso agudo en la saturación de oxígeno, taquicardia, disminución de ruidos respiratorios, presencia de esputo rosado, dificultad respiratoria, y enfisema subcutáneo entre otros. Los signos clínicos expuestos con anterioridad pueden presentarse hasta 48 horas después del evento explosivo.,

Por el mecanismo primario se produce una pérdida abrupta de la membrana alveolar, llevando a neumotórax, y si compromete estructuras vasculares, hemotórax; también es frecuente el edema pulmonar secundario al proceso inflamatorio y la vasodilatación. ${ }^{10}$

Es importante tener en cuenta que una de las causas de gran mortalidad en el trauma de tórax asociado a explosiones en espacios confinados es la embolia aérea, evento del cual se desconoce la incidencia. Esta entidad puede sospecharse cuando los pacientes presentan pérdida aguda de la visión, dolor torácico, déficit neurológico focal, livedo reticularis, petequias faríngeas y hemoptisis. $^{8}$

Todo paciente con trauma torácico debe contar con radiografía de tórax, estudio que acompañado de hallazgos clínicos es útil para identificar la presencia de compromiso aerodigestivo, hemotórax, neumotórax, fracturas costales, lesión de grandes vasos o cardiaca. En caso de presentar alguna de dichas lesiones debe procederse al manejo específico de la lesión. En caso de presentar falla respiratoria está indicada la ventilación con presión positiva. ${ }^{1,8}$ En los escenarios de guerra los soldados tienen chalecos antibalas que disminuyen la incidencia de trauma penetrante sin embargo el material propelido por la onda explosiva puede llevar a causar trauma contuso con fracturas costales, y contusión pulmonar por la transferencia de energía. ${ }^{1}$ En caso de que el paciente presente indicación de toracotomía, Se deben evitar los abordajes posterolaterales y laterales ya que dificultan un adecuado acceso al abdomen en caso de hacerse necesario durante el procedimiento. ${ }^{1}$ El daño cardíaco es usualmente causado por el mecanismo secundario. Se presenta como contusión miocárdica, embolia aérea, infarto agudo, arritmias, taponamiento cardiaco, hemorragia subintimal, inestabilidad hemodinámica y hematomas intramurales, por otro lado las laceraciones causadas por proyectiles son causa frecuente de taponamiento cardiaco y muerte. Es muy importante reconocer rápidamente estas heridas ya que pueden comprometer función de bomba y con esto llevar a la muerte. 8,10

El trauma de abdomen en estos pacientes es de mal pronóstico; con una supervivencia menor al 10\%. Este está presente en aproximadamente el $34 \%$ de los casos de pacientes con muerte inmediata en el evento. ${ }^{5}$ Similar a lo que ocurre en el tórax, aquellos combatientes que usan chalecos antibalas pueden presentar trauma contuso de abdomen con compromiso de órganos duros como hígado, bazo y riñones de allí que el la ultrasonografía de trauma FAST desempeña un rol esencial en el trauma por onda explosiva tanto en pacientes hemodinámicamente estables como inestables ya que un hallazgo positivo en un paciente estable indica la necesidad de explorar la cavidad. En la cirugía de guerra el paciente con trauma abdominal inestable y el paciente con trauma penetrante de abdomen tienen indicación quirúrgica de entrada; y debe considerarse un procedimiento diagnóstico y terapéutico dentro de un protocolo de control de daños controlando el sangrado y la infección para evitar la triada de la muerte., ${ }^{1,8,19-21}$

Otros estudios complementarios a realizar pueden ser la radiografía simple de abdomen y la tomografía axial computada de tórax, abdomen y pelvis; sin embargo el diagnóstico inicial es clínico y pueden manifestarse con un amplio espectro de signos y síntomas que van desde el paciente asintomático, pasado por el cuadro de abdomen agudo hasta llegar al abdomen no valorable. ${ }^{2,8}$

Una de las grandes dificultades del trauma abdominal secundario a explosivos es el conocer cuáles se van a perforar y cuáles no. ${ }^{8}$ Se ha descrito el uso de videocapsula endoscópica en el trauma abdominal por mecanismo primario con el objetivo de identificar perforaciones agudas o cambios en la mucosa que puedan predecir la perforación espontánea tardía ${ }^{2,8}$

En general los órganos intra-abdominales más frecuentemente afectados en el trauma por onda expansiva son el intestino delgado (48\%), el bazo (21.5\%) riñón (20\%), e hígado (15.3\%). ${ }^{1}$ Los órganos duros con frecuencia sufren ruptura, hemorragia, isquemia y necrosis; lo que se relaciona con trauma de alto impacto y poca distancia del lugar de detonación.

Estudios recientes recomiendan realizar la reconstrucción del tracto gastrointestinal en un segundo tiempo dado el estado catabólico y la pérdida de la microcirculación secundaria al trauma. ${ }^{8,18}$ La probabilidad de falla de la anastomosis al ser realizada en el primer momento quirúrgico es superior al $30 \%{ }^{1,8}$ Debe realizarse una una derivación entérica como una colostomía provisional buscando realizar un reconstrucción gastrointestinal a futuro. ${ }^{8}$ Aquellas Secciones de vísceras perforadas deben ser resecas ya que tienen riesgo de isquemia y necrosis por daño microvascular. ${ }^{1}$

Las explosiones pueden llevar a pérdida de la pared abdominal y torácica lo que se relaciona con una alta mortalidad; en caso de no causar la muerte usualmente requieren de una reconstrucción compleja., ${ }^{1,18}$ Dennis et al. proponen una clasificación para la perdida de pared abdominal, esta se describe en la Tabla $1 .{ }^{19}$ Este tipo de lesiones pueden llevar a una mecánica ventilatoria ineficiente. Ante este escenario se han propuesto múltiples estrategias como el uso de colgajos, injertos, mallas y terapia de presión negativa incluso en escenarios de contaminación severa., ${ }^{19}$

En cuanto al manejo del cierre de pared abdominal en algunos escenarios se hace imposible en un primer momento ya que la respuesta inflamatoria puede llevar a distensión de asas

Tabla 1: Grados de lesión de pared abdominal de Dennis et al. ${ }^{19}$

\begin{tabular}{ll}
\hline Grado & Definición \\
\hline II & $\begin{array}{l}\text { Contusión de tejido celular subcutáneo } \\
\text { Hematoma a nivel de la porción muscular } \\
\text { de la pared abdominal } \\
\text { III }\end{array}$ \\
IV & $\begin{array}{l}\text { Disrupción de un solo un músculo de la } \\
\text { pared abdominal }\end{array}$ \\
V & $\begin{array}{l}\text { Disrupción completa de la porción muscular } \\
\text { de la pared abdominal }\end{array}$ \\
& $\begin{array}{l}\text { Disrupción completa de la porción muscular } \\
\text { de la pared abdominal asociada a } \\
\text { VI }\end{array}$ \\
& $\begin{array}{l}\text { Disrupción completa de la porción } \\
\text { muscular de la pared abdominal asociada a } \\
\text { evisceración }\end{array}$ \\
\hline
\end{tabular}


y síndrome compartimental dificultando el cierre del abdomen. Como respuesta a ello Pengelly et al. publican en 2018 un estudio en donde de una cohorte de 155 pacientes, de los cuales el 51.3\% fueron candidatos para cierre primario y el $48.7 \%$ restante fue sometido a cierre temporal. Del segundo grupo 2 fallecieron, 2 fueron manejados con bolsa de Bogotá y los otros 66 fueron llevados terapia de presión negativa logrando el cierre de la pared en el $94 \%$ de los casos. Dicho estudio concluye que el cierre secuencial mejora la posibilidad de una eventual reconstrucción de la pared abdominal sin mejorar la incidencia de hernia incisional ya que no hubo diferencia estadísticamente significativa entre el el grupo de cierre primario y el cierre secuencial. ${ }^{22}$

Explosivos en el piso, como las minas antipersona, al ser detonadas, además de comprometer piernas y genitales, pueden ocasionar lesiones colorrectales comprometiendo la integridad del tracto gastrointestinal bajo. Ante dicha situación los cirujanos pueden optar por un reparo primario o por una derivación entérica en un primer momento quirúrgico para realizar una reconstrucción cuando el paciente no se encuentre en un estado catabólico. ${ }^{21,23-26}$ Glasgow et al. publican un estudio retrospectivo donde a partir de su experiencia en el manejo de trauma anal durante las guerras de Iraky Afganistán encuentran los siguientes resultados: el 76.1\% de las lesiones de ano y recto fueron secundarias a explosiones. El 78.2\% de estos pacientes requirieron derivación entérica en la mayoría de los casos por trauma severo de otras estructuras abdominopélvicas asociadas. El $30.3 \%$ de los pacientes requirieron colostomías permanentes, siendo predictores fuertes de la necesidad de esta la presencia de lesiones gastrointestinales severas $(p=0.009)$, ligadura de arteria hipogástrica $(p=0.05)$ y fractura pélvica $(p=0.06) .{ }^{24}$

Watson et al. en un estudio retrospectivo identificaron 867 militares con lesiones colorrectales en los combates de Irak y Afganistán durante un periodo de 8 años. La incidencia total de colostomía fue de $37 \%$ siendo la mayor causa de derivación entérica la lesión rectal (56\%), seguidas de lesiones de hemiabdomen izquierdo y derecho (41 y $21 \%$ respectivamente). También como resultado se vio una correlación entre un ISS (injury severity score) mayor a 16 y la necesidad de cirugía de control de daños con una probabilidad mayor de requerir una colostomía. ${ }^{27}$

En cuanto a lesiones genitourinarias asociadas a explosiones es necesario mencionar que las estructuras más frecuentemente comprometidas son el escroto (39\%), testículos (36\%), pene (20\%) y uretra (5\%). Estas lesiones con frecuencia se encuentran asociadas a compromiso de estructuras vitales y por si mismas usualmente no comprometen la vida del paciente, de allí que sea necesario priorizar la atención hacia otras heridas potencialmente mortales. $^{25}$

Algunos pacientes con antecedente de trauma pueden presentar neumoperitoneo como hallazgo incidental sin presentar lesión visceral o violación de la pared abdominal como lo reportan Bowley et al., en dos casos. Algunas teorías apuntan a que esta situación es consecuencia de lesiones pulmonares que llevan a la presencia de aire en el mediastino, mismo aire que posteriormente migra hacia el abdomen por medio de aperturas anatómicas como el hiato esofágico, y aórtico. ${ }^{28}$

Aunque es poco usual, se ha descrito la ruptura diafragmática tardía posterior a trauma por onda explosiva como lo reporta Weerink et al. en un caso de un paciente que un año después de la exposición al evento explosivo. ${ }^{29}$

Las lesiones en extremidades son frecuentes en aquellos pacientes que sobreviven al evento inicial. ${ }^{1}$ A pesar que de que estas son las más evidentes el cirujano no debe distraerse ya que es probable que la amputación no sea la herida que está comprometiendo la vida. ${ }^{8}$

La mayoría de las lesiones en extremidades son mediadas por el mecanismo de lesión secundario aunque también es frecuente el mecanismo cuaternario con fracturas conminutas y síndrome compartimental posterior. ${ }^{1,8}$

El daño de extremidades asociado a explosivos usualmente compromete tejidos blandos y duros. Es por ello que la meta principal es hacer el control de daños para preservar la longitud del miembro, la perfusión del mismo y el máximo tejido viable para lograr un adecuado cubrimiento de la herida. En el caso de que sea necesaria una amputación esta debe realizarse lo más distal posible e idealmente por un especialista en ortopedia., ${ }^{1,8}$

Los signos duros y blandos de trauma vascular deben evaluarse con suma cautela, la extremidad debe lavarse y desbridar completamente mientras se hace control hemostático ya sea con anastomosis o ligadura de vasos dependiendo del caso. ${ }^{1,8,30}$ Así lo recomiendan Tresson et al. en 2017 cuando publican en Annals of Vascular Surgery su experiencia en el manejo de trauma vascular en los ataques terroristas de París en 2015 donde 351 personas fueron heridas pero solo 3 presentaron trauma vascular periférico como consecuencia a su exposición a elementos explosivos. ${ }^{7}$ Van Dongen et al. en su experiencia como cirujanos militares Holandeses en Afganistán, atendieron un total de 194 con trauma vascular, de los cuales 99 tuvieron compromiso vascular periférico, demostrando una sobrevida superior en este grupo vs el grupo de trauma vascular central. La amputación de miembro fue necesaria en el $86 \%$ de los casos con lesión de miembros inferiores y en el $53 \%$ de las lesiones de miembros superiores. El reparo de estructuras vasculares fue posible en el $46 \%$ de los casos en los cuales fue posible salvar la extremidad con una tasa de éxito del $69.2 \% .^{31}$ Los explosivos con frecuencia ocasionan lesiones que requieren un control de daños vascular muy proximal para evitar la exanguinación; escenario que puede llevar a amputaciones altas. Esto es relevante ya que la mortalidad en lesiones con amputación traumática transfemoral bilateral lleva a mortalidad en el $90 \%$ de los casos. ${ }^{32}$

En pacientes con sangrado arterial severo se ha utilizado el REBOA "resuscitative endovascular balloon occlusion of the aorta" para el control de hemorragia evidenciando buenos resultados al mantener las presión arterial central, la perfusión de órganos vitales y la sobrevida de pacientes con hemorragias pélvicas severas como se ha demostrado en modelos humanos y animales. ${ }^{33}$

Como es de resaltar la mayoría de hallazgos y técnicas innovadoras ha surgido por el ámbito bélico que rodea a la humanidad, así como lo muestra Asensio et al., el trauma vascular es uno de los campos que más han evolucionado, y es de reconocer que los cirujanos que lidian con lesiones vasculares se han enfrentado a un camino difícil y arduo, ${ }^{34}$ así como lo decía William Stewart Halsted en 1912: "Una de las principales fascinaciones en la cirugía es el manejo de los vasos lesionados."

Las nuevas técnicas endovasculares junto con el uso de quirófanos híbridos eventualmente marcaran la diferencia en el tratamiento de lesiones vasculares, aunque en la actualidad sus funciones aún siguen en estudio, aunque hasta el dia de hoy se ve poca utilidad en la cirugía de guerra.

El papel del control de daños en las extremidades vasculares seguirá desempeñando un papel importante ya que ha tenido resultados exitosos con el uso de derivaciones vasculares temporales las cuales se han reportado tanto en el ámbito civil como militar. ${ }^{34}$ 
En cuanto al manejo de infección se debe recalcar que el lavado debe ser exhaustivo ya que se considera un tejido altamente contaminado; $y$ debe realizarse con gluconato de clorhexidina para posteriormente hacer profilaxis antitetánica. ${ }^{8}$ La principal causa de morbimortalidad hospitalaria no aguda del herido en combate, exceptuando las secuelas inmediatas del desangramiento, es la infección, ${ }^{3}$ sobre todo en aquellos pacientes que son penetrados por elementos repelidos por la detonación. Los microorganismos más frecuentemente relacionados con este tipo de infecciones son Estreptococos, Estafilococos, Enterobacterias (siendo la E. coli las más frecuente), y Pseudomonas. ${ }^{33}$

En nuestro medio se ha reportado que las infecciones asociadas a la guerra son causadas principalmente por bacterias Gram negativas. En general, E. coli es la primera bacteria causante de infección (35\%). Es indispensable conocer la resistencia local de la bacteria a los antibióticos, para determinar la mejor selección terapéutica. ${ }^{3}$ En el análisis prospectivo de 2010 a 2012, en el que se incluyeron 5.506 pacientes con cultivos positivos para $E$. coli, el porcentaje de resistencia a ampicilina-sulbactam, a trimetoprimsulfametoxazol y a ciprofloxacina, fue cercano a $40 \%$, por lo que el antibiótico se debe seleccionar excluyendo los anteriores y con base en el resultado del antibiograma. ${ }^{3}$ Hospenthal et al. en 2008 publican una guía para la prevención de la infección en el escenario bélico en donde recomiendan que en las primeras 3 horas debe iniciarse manejo antibiótico. Cuando la lesión es a nivel de extremidades debe iniciarse moxifloxacina o levofloxacina y en caso de lesión abdominal, choque e inestabilidad hemodinámica está indicado el uso de ertapenem como primera línea o cefoxitin endovenoso como segunda opción. ${ }^{29,35}$

\section{Conclusión}

El trauma por onda explosiva puede llevar a comprometer la vida por lo cual el cirujano general que se enfrente a esta situación debe tener un conocimiento pleno sobre los mecanismos fisiopatológicos de las lesiones, los patrones y las estructuras usualmente comprometidas para dar un manejo óptimo a este tipo de lesiones. Es necesario un abordaje multidisciplinario viendo al paciente en toda sus esferas (biológica, psicológica y sociocultural) para llegar a los mejores resultados posibles.

\section{References}

1. Plurad D. Blast injury. Mil Med 2011;176:276-282. DOI: 10.7205/ MILMED-D-10-00147.

2. Owers C, Morgan JL, Garner JP. Abdominal trauma in primary blast injury. Br J Surg 2011;98(2):168-179. DOI: 10.1002/bjs.7268.

3. Camargo J, Pérez LE, Franco C, et al. "Plan pantera", trauma Militar en Colombia. Rev Colomb Cir 2014;29:293-304.

4. Popivanov G, Mutafchiyski VM, Belokonski El, et al. A modern combat trauma. J R Army Med Corps 2014;160(1):52-55. DOI: 10.1136/jramc2013-000132.

5. Turégano-Fuentes F, Pérez-Diaz D, Sanz-Sánchez M, et al. Abdominal blast injuries: different patterns, severity, management, and prognosis according to the main mechanism of injury. Eur J Trauma Emerg Surg.2014;40(4):451-460. DOI: 10.1007/s00068-014-0397-4.

6. Hoencamp R, Huizinga EP, van Dongen TT, et al. Impact of explosive devices in modern armed conflicts: in-depth analysis of dutch battle casualties in southern Afghanistan. World J Surg 2014;38(10): 2551-2557. DOI: 10.1007/s00268-014-2645-5.

7. Tresson P, Touma J, Gaudric J, et al. Management of vascular trauma during the Paris terrorist attack of November 13, 2015. Ann Vasc Surg 2017;40:44-49. DOI: 10.1016/j.avsg.2016.09.011.
8. Gale SC, Shiroff AM, Donovan CM, et al. Medical management at the health care facility. Ann Emerg Med 2017;69(1S):S36-S45. DOI: 10.1016/j.annemergmed.2016.09.009.

9. Poon H, Morrison JJ, Apodaca AN, et al. The UK military experience of thoracic injury in the wars in Iraq and Afghanistan. Injury 2013;44(9):1165-1170. DOI: 10.1016/j.injury.2013.01.041.

10. McLean MM, Demijohn B, Wallen T, et al. An 11-year-old who suffered multiple traumatic injuries secondary to a house explosion. Air Med J 2017;36(4):151-155. DOI: 10.1016/j.amj.2017.05.007.

11. Hakimoglu S, Karcıoglu M, Tuzcu K, et al. Assessment of the perioperative period in civilians injured in the syrian civil war. Braz J Anesthesiol 2015;65(6):445-449. DOI: 10.1016/j.bjan.2014.03.003.

12. Han G, Wang Z, Wang J, et al. Characteristics and mechanisms of cardiopulmonary injury caused by mine blasts in shoals: a randomized controlled study in a rabbit model. PLoS One 2013;8(12):e81310. DOI: 10.1371/journal.pone.0081310.

13. Ramman S, Al-Rubayee H. Every wound counts: a case of undiscovered wound in mons pubis resulting in a chronic foreign body granuloma of the abdominal wall. Int Wound J 2014;11(6):579-580. DOI: 10.1111/ iwj.12303.

14. Maqsood R, Rasikh A, Abbasi T, et al. Pattern of injuries seen in mass casualties in terrorist attacks in Baluchistan, Pakistan--a three years experience. J Ayub Med Coll Abbottabad 2015;27(4):858-860.

15. Schoenfeld AJ, Dunn JC, Bader JO, et al. The nature and extent of war injuries sustained by combat specialty personnel killed and wounded in Afghanistan and Iraq, 2003-2011. J Trauma Acute Care Surg 2013;75(2):287-291. DOI: 10.1097/TA.0b013e31829a0970.

16. Glaser J, Vasquez M, Cardarelli C, et al. Ratio-driven resuscitation predicts early fascial closure in the combat wounded. J Trauma Acute Care Surg 2015;79(4 Suppl 2):S188-S192. DOI: 10.1097/ TA.0000000000000741.

17. Van Vugt R, Keus F, Kool D, et al. Selective computed tomography (CT) versus routine thoracoabdominal CT for high-energy blunttrauma patients. Cochrane Database Syst Rev 2013;12:CD009743. DOI: 10.1002/14651858.CD009743.pub2.

18. Mitchell TA, Hutchison T, Becker TE, et al. Nontherapeutic laparotomy in american combat casualties: a 10-year review. J Trauma Acute Care Surg 2014;77(3 Suppl 2):S171-S175. DOI: 10.1097/ TA.0000000000000367.

19. Dennis RW, Marshall A, Deshmukh H, et al. Abdominal wall injuries occurring after blunt trauma: incidence and grading system. Am J Surg 2009;197(3):413-417. DOI: 10.1016/j.amjsurg.2008.11.015.

20. Mitchell TA, Lauer CL, Aden JK, et al. Short-term outcomes and complications of damage control and definitive laparotomy in deployed combat environments: 2002 to 2011. Mil Med 2016;181(3):277-282. DOI: 10.7205/MILMED-D-14-00726.

21. Smith IM, Beech ZK, Lundy JB, et al. A prospective observational study of abdominal injury management in contemporary military operations: damage control laparotomy is associated with high survivability and low rates of fecal diversion. Ann Surg 2015;261(4):765-773. DOI: 10.1097/SLA.0000000000000657.

22. Pengelly S, Berry JEA, Herrick SE, et al. Outcome of open abdominal management following military trauma. Br J Surg 2018;105(8):980986. DOI: 10.1002/bjs.10813.

23. Bortolin M, Baldari L, Sabbadini MG, et al. Primary repair or fecal diversion for colorectal injuries after blast: a medical review. Prehosp Disaster Med 2014;29(3):317-319. DOI: 10.1017/S1049023X14000508.

24. Glasgow SC, Heafner TA, Watson JD, et al. Initial management and outcome of modern battlefield anal trauma. Dis Colon Rectum 2014;57(8):1012-1018. DOI: 10.1097/DCR.0000000000000141.

25. Banti M, Walter J, Hudak S, et al. Improvised explosive device-related lower genitourinary trauma in current overseas combat operations. J Trauma Acute Care Surg 2016;80(1):131-134. DOI: 10.1097/ TA.0000000000000864

26. O'Donnell MT, Greer LT, Nelson J, et al. Diversion remains the standard of care for modern management of war-related rectal injuries. Mil Med 2014;179(7):778-782. DOI: 10.7205/MILMED-D13-00533. 
27. Watson JD, Aden JK, Engel JE, et al. Risk factors for colostomy in military colorectal trauma: a review of 867 patients. Surgery 2014;155(6):1052-1061. DOI: 10.1016/j.surg.2014.01.010.

28. Bowley DM, Gillingham S, Mercer S, et al. Pneumoperitoneum without visceral trauma: an under-recognised phenomenon after blast injury? J R Army Med Corps 2013;159(4):312-313. DOI: 10.1136/jramc-2013000066.

29. Weerink LB, Ten Duis K, Van der Velde D, et al. Delayed presentation of diaphragmatic rupture after blast injury. J R Army Med Corps 2013;159(4):304-306. DOI: 10.1136/jramc-2013-000068.

30. Hathaway E, Glaser J, Cardarelli C, et al. Exploratory laparotomy for proximal vascular control in combat-related injuries. Mil Med 2016;181(5 Suppl):247-252. DOI: 10.7205/MILMED-D-15-00155.

31. van Dongen TT, Idenburg FJ, Tan EC, et al. Combat related vascular injuries: dutch experiences from a role 2 MTF in Afghanistan. Injury 2016;47(1):94-98. DOI: 10.1016/j.injury.2015.08.030.
32. Poon H, Morrison JJ, Clasper JC, et al. Use and complications of operative control of arterial inflow in combat casualties with traumatic lower-extremity amputations caused by improvised explosive devices. J Trauma Acute Care Surg 2013;75(2 Suppl 2):S233-S237. DOI: 10.1097/TA.0b013e318299d99d.

33. Fares $\mathrm{Y}$, El-Zaatari M, Fares J, et al. Trauma-related infections due to cluster munitions. J Infect Public Health 2013;6(6):482-486. DOI: 10.1016/j.jiph.2013.05.006.

34. Asensio JA, Petrone P, Pérez-Alonso A, et al. Contemporary wars and their contributions to vascular injury management. Eur J Trauma Emerg Surg 2015;41(2):129-142. DOI: 10.1007/s00068-0140430-7.

35. Hospenthal DR, Murray CK, Andersen RC, et al. Guidelines for the prevention of infection after combat-related injuries. J Trauma 2008;64(3 Suppl):S211-S220. DOI: 10.1097/TA.0b013e318163 c421. 